\title{
Adaptive NK cells undergo a dynamic modulation in response to human cytomegalovirus and recruit T cells in in vitro migration assays
}

\author{
Débora Basílio-Queirós $\mathbb{D}^{1 凶}{ }^{凶}$, Letizia Venturini ${ }^{1,2}$, Susanne Luther-Wolf ${ }^{1}$, Elke Dammann ${ }^{1}$, Arnold Ganser ${ }^{1}$, Michael Stadler ${ }^{1}$, \\ Christine S. Falk ${ }^{2,3}$ and Eva M. Weissinger (D) ${ }^{1,2}$
}

(C) The Author(s) 2022

\begin{abstract}
Human cytomegalovirus (HCMV) reactivation remains a relevant complication after hematopoietic stem cell transplantation (HSCT) despite the great progress made in prophylaxis and treatment. Adaptive Natural Killer (NK) cells undergo a persistent reconfiguration in response to HCMV reactivation however, the exact role of adaptive NK cells in HCMV surveillance is currently unknown. We studied the relationship between HCMV reactivation and adaptive NK cells in 70 patients monitored weekly until day +100 after HSCT. Absolute cell counts of adaptive NK cells increased significantly after resolution of HCMV-reactivation compared to patients without reactivation. Patients with HCMV-reactivation had an early reconstitution of adaptive NK cells ("Responders") and had mainly a single reactivation (75\% Responders vs $48 \%$ Non-Responders). Adaptive NK cells eliminated HCMV-infected human foreskin fibroblasts (HFF) in vitro and recruited T cells in an in vitro transwell migration assay. An extensive cytokine/ chemokine panel demonstrated strongly increased secretion of CXCL10/IP-10, IFN-a, IL-1a, IL-1 $\beta$, IL-5, IL-7 and CCL4. Thus, adaptive NK cells may control viral spread and T cell expansion and survival during HCMV-reactivation. Taken together, we have demonstrated the potential of adaptive NK cells in the control of HCMV reactivation both by direct cytotoxicity and by recruitment of other immune cells.
\end{abstract}

Bone Marrow Transplantation (2022) 57:712-720; https://doi.org/10.1038/s41409-022-01603-y

\section{INTRODUCTION}

Allogeneic hematopoietic stem cell transplantation (HSCT) remains a curative treatment for malignant and even some non-malignant hematological disorders [1]. Despite the great progress made in the reduction of toxicities of conditioning regimens and improvements in immunosuppression, HSCT is still hampered by severe complications. Next to relapse and graft-versus-host-disease infections, especially viral reactivations, lead to a significant increase of morbidity and even mortality [2-5]. HCMV has a prevalence of $50-90 \%$ in the human population, depending on age, geographical and socioeconomic factors [6]. After primary infection HCMV establishes a life-long latent infection [7]. Reactivation of HCMV can occur in up to $70 \%$ of seropositive recipients $(R+)$ even when transplanted from HCMV-seropositive donors ( $D+$ ). In addition to the previously mentioned increases in morbidity and mortality rates, HCMV-reactivations (HCMV-R) also lead to a reduction of quality of life due to prolonged hospitalization $[8,9]$.

While the role of T cells in the control of HCMV-R is quite accepted and understood to date, Natural Killer (NK) cells may also play an important role in immune reconstitution and surveillance. NK cells are innate lymphocytes well described by their ability to kill virally infected or malignant cells. NK cells are the main lymphocytic population to reconstitute early after HSCT [10]. The crucial role of
NK cells in viral infection control is associated not only to the early reconstitution of this cell population (when other major players of the immune response are still lacking) but also by their particular response to different viruses. For instance, different viruses will promote the expansion of different NK cell subpopulations and trigger different cytotoxic/regulatory approaches. These tailored responses have been described for several viral infections, included among others, HCMV, Hepatitis B virus, Epstein-Barr virus and Human Immunodeficiency virus. This has been discussed in more length by Zuo and Zhao [11]. Furthermore, a prospective study by a Drylewicz et al. suggests that the rapid reconstitution of $\mathrm{CD} 4^{+} \mathrm{T}$ cells and NK cells (rather than the cytotoxic T cell population), is associated with the absence of HCMV-R after HSCT [12].

More recently, a subpopulation of NK cells expressing NKG2C has been identified and is increased in HCMV-seropositive healthy individuals. In addition, $\mathrm{NKG}_{2} \mathrm{C}^{+}$cells expand upon co-cultivation with HCMV-infected fibroblasts [13, 14]. This NK cell subpopulation preferentially expresses CD57 and shows downregulation of activating markers such as NKp30 and NKp46 compared to conventional NK cells $[15,16]$. Furthermore, HCMV infection shapes the NK cell repertoire in humans. Murine studies demonstrated that adoptively transferred adaptive NK cells protected against MCMV infection/ reactivation [13, 17]. Thus,

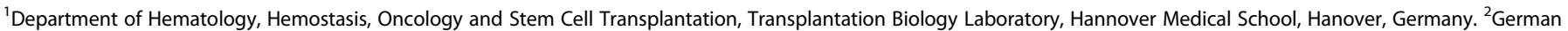
Center for Infection Research (DZIF), Site Hannover-Braunschweig, Brunswick, Germany. ${ }^{3}$ Hannover Medical School, Institute of Transplant Immunology, Hanover, Germany.

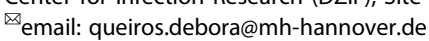

Received: 7 April 2021 Revised: 17 January 2022 Accepted: 27 January 2022

Published online: 17 February 2022 
these NK cells may play an adaptive-like role and are called memory-like or adaptive NK cells. Adaptive NK cells are detected in healthy HCMV-seropositive individuals, but are quite heterogeneous which may be due to the peptide-specific recognition of HCMV strains [18]. It is still unknown how adaptive NK cells are able to specifically respond to HCMV but their potential role in the immune reconstitution after HSCT is of great interest [19]. In addition, adaptive NK cells exhibit characteristics belonging to both the innate and adaptive branches of the immune system. For this reason, their possible role in bridging these two sides is also of great clinical interest. To date, the interaction of the innate and the adaptive immune system are of great interest. Several publications have shown that both NK and T cells need to be expanding to protect against recurrent $\mathrm{CMV}$ reactivations. Here we show that adaptive NK cells secrete a number of cytokines involved in T cell expansion and using migration assays we show that $T$ cells migrate toward only the stimulated adaptive NK cells.

In the present study, we analyzed the reconstitution, expansion and function of adaptive NK cells in the context of HCMV reactivation in HCMV-seropositive donors and/or recipients pairs after HSCT.

\section{RESULTS}

\section{Adaptive NK cells expand and persist during and after HCMV reactivation}

Peripheral blood (PB) samples from $70 \mathrm{HCMV-seropositive} \mathrm{patient/}$ donor pairs were examined for differential reconstitution of adaptive NK cells weekly up to day +100 after HSCT. Clinical and demographic data of the patients are summarized in Table 1. HCMV-serostatus of recipient and donor, number of patients with HCMV-R and the median day of HCMV-R are summarized in Table 2.

Patients with and without HCMV-R were analyzed as matched pairs according to days after HSCT. Figure 1a shows that although adaptive NK counts are low prior to HCMV-R, a dynamic modulation of absolute counts is seen during HCMV-R. An average 1.32 fold expansion of adaptive NK cells was observed in patients after HCMV$R$ compared to an average 0.92 fold increase in patients without HCMV-R (Fig. 1d). Remarkably, after resolution of the HCMV-R, these counts were significantly increased to an average 5.3 fold expansion $(p<0.01)$. Furthermore, after the resolution of HCMV-R, absolute counts of mature adaptive NK cells (CD57 ${ }^{+}$adaptive NK cells) were significantly increased in patients with HCMV-R compared to patients without HCMV-R (Fig. 1b). The comparison of absolute counts of total NK cells in patients with and without HCMV-R at the same time-points after HSCT is shown in Fig. 1c. The average-fold expansion of adaptive NK cells was independent of the increase of total NK cells in PB and thus not a consequence of the time after HSCT. Interestingly, overall NK cell counts were not influenced by the expansion of adaptive NK cells after HCMV-R.

\section{HCMV-R promotes a long-lasting increase in both HCMV-CTLs} and adaptive NK cells

Next, the relation between the expansion of adaptive NK cells and HCMV-specific cytotoxic T lymphocytes (HCMV-CTLs) in response to HCMV-R was investigated by tetramer staining (Fig. 2). Whole blood was analyzed for the presence of HCMV-CTLs at the time points indicated in the methods section. The number of tetramers used for each patient was dependent on the HLA typing of both donor and recipient and the commercial availability of HCMV-tetramers for the individual HLA alleles. As shown before (Borchers et al.) the expansion of CMV-CTLs after HCMV-R is an indicator for immunity against recurrent reactivations, therefore the sum of all $\mathrm{CD}^{+} \mathrm{Te}-$ tramer $^{+} \mathrm{T}$ cells can be used to assess immunity.

HCMV-CTLs expanded in response to HCMV-R in most patients, while adaptive NK cells expanded to a lesser degree during reactivation. Interestingly, adaptive NK cells expanded significantly when HCMV-R had been resolved and the T cell compartment began to contract $(p<0.05)$.
Table 1. Clinical and demographic parameters of patients included in the cohort.

\begin{tabular}{|l|l|}
\hline Age & $\begin{array}{l}\text { Median 58 } \\
\text { (range 19-73) }\end{array}$ \\
\hline Disease & $46(65.7 \%)$ \\
\hline Acute (AML, ALL, sAML, s/tAML) & $14(20 \%)$ \\
\hline $\begin{array}{l}\text { Chronic (MDS, MPS, CML, CLL, MM, } \\
\text { MD/MPN) } \\
\text { Lymphoma (NHL, HD) }\end{array}$ & $6(8.6 \%)$ \\
\hline Non-malignant (AA, PNH) & $4(5.7 \%)$ \\
\hline Conditioning & \\
\hline Myeloablative & $12(17.1 \%)$ \\
\hline RIC & $58(82.9 \%)$ \\
\hline GvHD-Prophylaxis & \\
\hline CSA/MMF & $54(77.1 \%)$ \\
\hline CSA/MTX & $12(17.1 \%)$ \\
\hline Other & $4(5.7 \%)$ \\
\hline HLA-match & \\
\hline Matched & $56(80 \%)$ \\
\hline Mismatched & $14(20 \%)$ \\
\hline Donor & $20(28.6 \%)$ \\
\hline Related & $50(71.4 \%)$ \\
\hline Unrelated & $29(41.4 \%)$ \\
\hline Gender & $41(58.6 \%)$ \\
\hline Female & \\
\hline Male & \\
\hline
\end{tabular}

acute: $A M L$ acute myeloid leukemia, $A L L$ acute lymphatic leukemia, $s A M L$ secondary AML, chronic: MDS/MPS myelodysplastic/proliferative syndrome, CML chronic myeloid leukemia, CLL chronic lymphatic leukemia, lymphoma: NHL non-Hodgkin lymphoma, HD Hodgkin disease, MM multiple myeloma; non-malignant: (S)AA severe or very severe aplastic anemia, $P N H$ paroxysmal nocturnal hematouria, myeloablative conditioning: $R I C$ reduced intensity conditioning, $P B S C$ peripheral blood stem cells, $B M$ bone marrow, $C B$ cord blood, ATG anti-thymocyte globulin, CSA cyclosporine $A$, MTX methotrexate, MMF mycophenolate motefil, other: MMF tacrolimus (FK506), or different combinations of immunosuppressants.

Table 2. HCMV-serostatus and patient groups.

$\begin{array}{ll}\text { CMV serostatus } & \text { Number of patients } \\ \mathrm{R}^{+} \mathrm{D}^{+} & 42 \\ \mathrm{R}^{+} \mathrm{D}^{-} & 18 \\ \mathrm{R}^{-} \mathrm{D}^{+} & 10 \\ \mathrm{CMV} \text { Reactivation } & 37(52.9 \%) \\ \mathrm{CMV} \text { Reactivation day post-transplantation } & 37 \text { (range 17-63) } \\ R^{+} D^{+} \text {recipient and donor are anti-cytomegalovirus lgG-positive, } R^{+} D^{-} \\ \text {recipient is anti-cytomegalovirus IgG-positive, } D^{-} \text {donor is IgG and IgM- } \\ \text { negative. }\end{array}$

\section{Adaptive NK cells degranulate and eliminate HCMV-infected cells}

The notable expansion of adaptive NK cells led to further investigations of their possible role in controlling HCMV-R. Sorted mature adaptive $\mathrm{NK}$ cells $\left(\mathrm{NKG} 2 \mathrm{C}^{+} \mathrm{CD} 57^{+}\right)$were expanded in vitro for 14 days, to obtain sufficient numbers of adaptive NK cells for functional assays. To determine the role of adaptive NK cells in the elimination of HCMV-infected cells, CD107a degranulation assay and the lactate dehydrogenase $(\mathrm{LDH})$ release assay were performed. Figure $3 \mathrm{~b}$ shows that degranulation of stimulated 

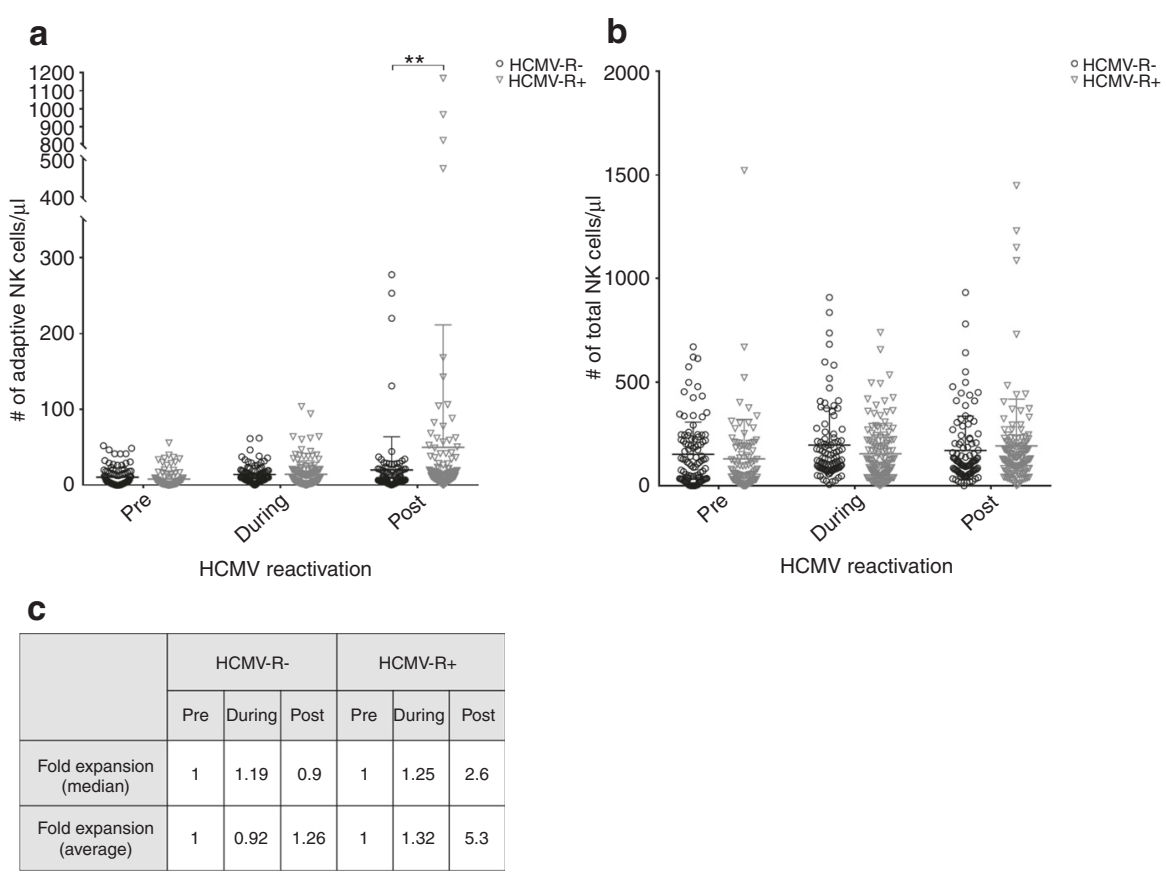

Fig. 1 Absolute counts of adaptive NK cells in patients with or without HCMV reactivation. Cell aggregates were excluded by FSC-H vs FSCA gating. SSC-A vs FSC-A gating discriminated between counting beads and lymphocytes. CD3, CD14 and 7AAD vs CD56 were used to discriminate NK cells from other lymphocytic populations. a Adaptive NK cells were selected as NKG2C-positive NK cells. Patients with HCMV reactivation ( $\mathrm{HCMV}-\mathrm{R}+$ ) were analyzed in matched days post-HSCT with patients without HCMV reactivation (HCMV-R-). Absolute counts were calculated according to the formula: Adaptive NK cells $/ \mu \mathrm{L}=\frac{\text { number of events in cell population }}{\text { number of events in absolute count bead region }} \times \frac{\text { number of known beads per test }}{\text { test volume }}$. A diagnosis of HCMV reactivation was made when 5 or more pp65 antigen-positive cells per $4 \times 10^{5}$ leukocytes were detected in the peripheral blood of a given patient. Resolution of HCMV reactivation was diagnosed after three consecutive negative pp65 antigen tests. b Absolute cell counts of unselected NK cells per $\mu \mathrm{l}$ of blood were calculated by the formula above. $\mathbf{c}$ The summary of the median and average-fold expansion of adaptive NK cells in patients without HCMV reactivation (HCMV-R-) and patients with HCMV-reactivation (HCMV-R+) is tabulated. Statistical analysis represents two-way ANOVA with Sidak's multiple comparison test. Mean and standard deviation are shown. $n=70 ;{ }^{* *} p<0.01$.

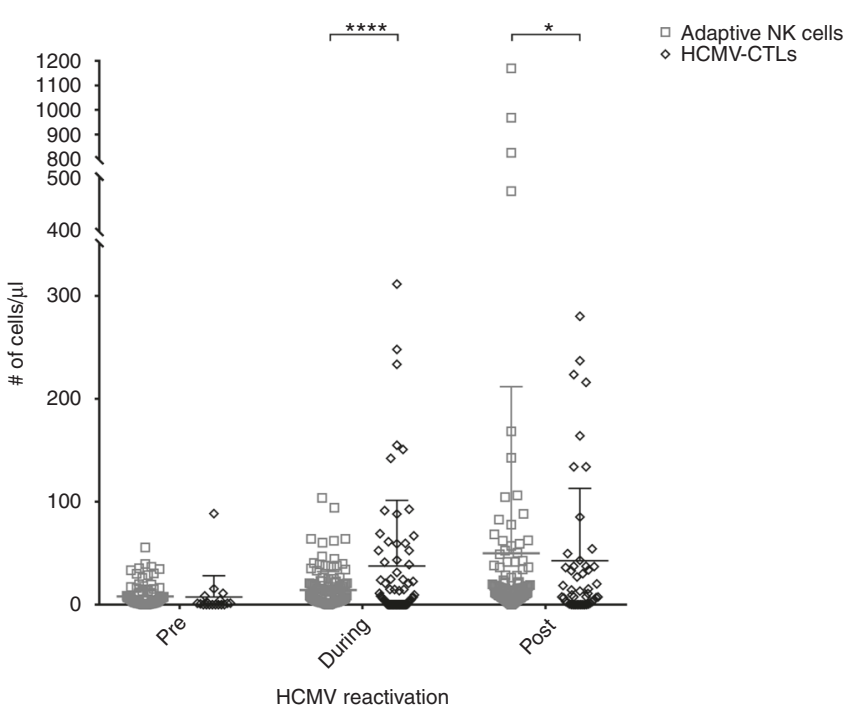

Fig. 2 Absolute cell counts of adaptive NK cells and HCMV-CTLs. Patients were monitored for adaptive NK cell and HCMV-CTLs reconstitution after HSCT. Absolute cells counts are shown. Absolute counts of HCMV-CTLs were calculated according to the formula: HCMV$\mathrm{CTLS} / \mu \mathrm{L}=\left(\%\right.$ specific tetramer ${ }^{\text {positive cells }}-\%$ control tetramer $\left.{ }^{\text {positive cells }}\right) \times$ absolute number of $\mathrm{CD}^{+}$cells. Statistical analysis represents two-way ANOVA with Sidak's multiple comparison test. Mean and standard deviation are shown. $n=37 ;{ }^{*} p<0.05 ;{ }^{* * * *} p<0.0001$. adaptive NK cells in response to HFF-TB40-BAC was significantly higher than that of adaptive NK cells cultured with uninfected HFF alone at the effector to target ratio of 1:1 $(p<0.01)$. Furthermore, Fig. $3 c$ shows a significant increase of $L D H$ release when adaptive NK cells were co-cultured with HFF-TB40-BAC compared to noninfected HFF $(p<0.001)$ indicating lysis of the target cells.

Adaptive NK cells recruit $\mathrm{T}$ cells in in vitro migration assays To address the role of adaptive NK cells in HCMV-R and to give a plausible explanation for the marked expansion of absolute cell counts after HCMV-R, we performed migration assays to test the capacity of adaptive NK cells to recruit T cells.

Autologous T cells were cultured in the upper chamber of a transwell system. Cells or chemokines were added to the lower chamber of the transwell system as described in the methods section. The fold migration under the different culture conditions was calculated. Adaptive NK cells cultured without target cells promoted an average 6-fold migration of T cells (Fig. 4C). Coculture of adaptive NK cells with HFF-TB40-BAC led to a striking increase of 28 -fold migration of $T$ cells. This was statistically significant compared to all other conditions tested, and also statistically significantly increased when compared to the migration of the T cells stimulated with the chemoattractant CCL21 used as positive control $(p<0.0001)$.

Adaptive NK cells secrete a number of activating cytokines/ chemokines in transwell assays

To address the excretion of cytokines and chemokines released under the different conditions in the transwell assay the cell-free supernatants were analyzed in a multiplex protein array quantifying 29 cytokines, chemokines and growth factors. Figure 5a shows 
a

Pre-sorting
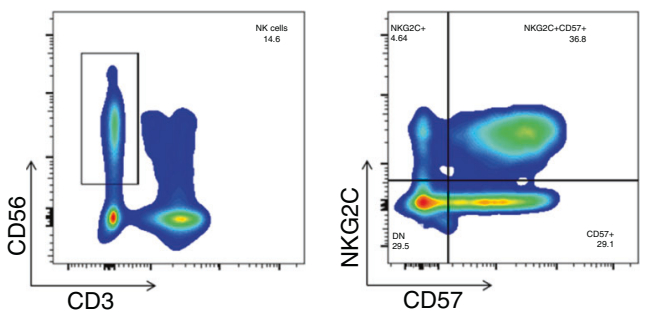

Post-sorting
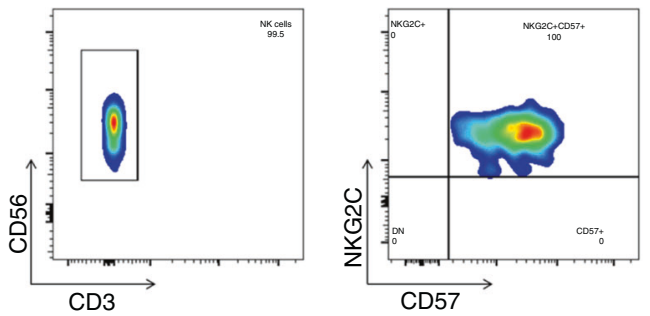

Post-expansion
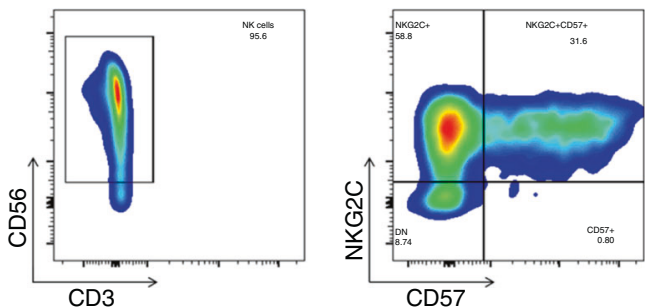

b
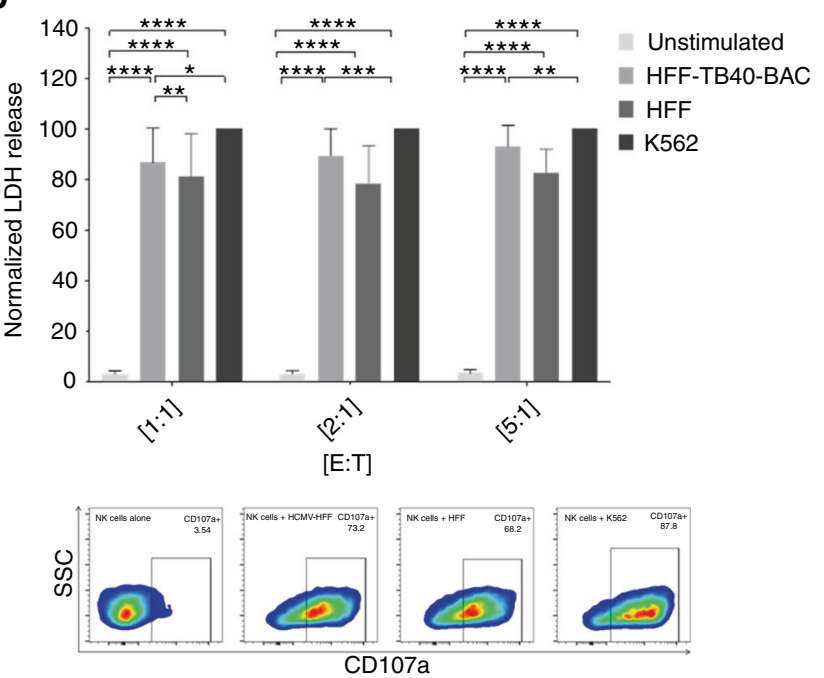

c

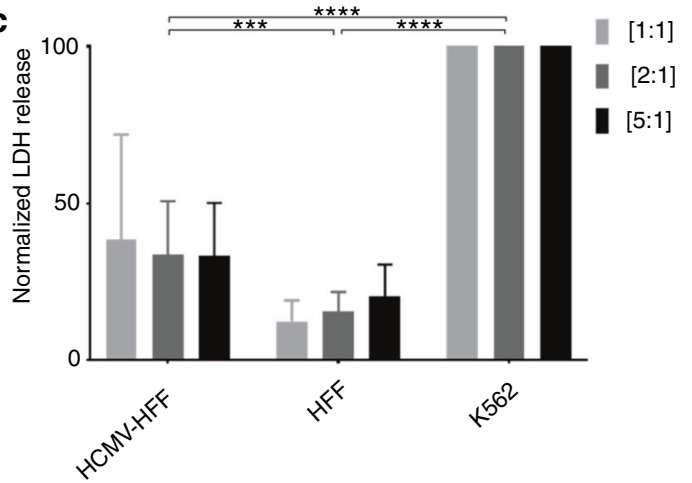

Fig. 3 Cytotoxic capacity of adaptive NK cells. In vitro expanded adaptive NK cells were co-cultured with uninfected HFF, HFF-TB40-BAC, or K562 cells at E:T ratios of 1:1; 2:1 and 5:1 as indicated. a Representative density plot of NK cells pre- and post-sorting and post-expansion. b Normalized percentage of CD107a staining and representative density plot of CD107a surface expression at an E:T ratio of 1:1. c The percentage of LDH release was normalized by setting the LDH release in response to K562 to $100 \%$. Statistical analysis represents two-way ANOVA with Tukey's multiple comparison. Mean and standard deviation are shown. $n=7 ;{ }^{*} p<0.05 ;{ }^{* *} p<0.01 ;{ }^{* * *} p<0.001 ;{ }^{* * * *} p<0.0001$.

cytokines and chemokines were excreted at significantly different concentrations when HCMV-stimulated adaptive NK cells were attracting $T$ cells to the lower chambers in vitro.

Eleven of 29 factors analyzed were significantly increased. Five were activating cytokines, such as interferon alpha (IFN-a), IL-1a, IL-1 $\beta$, IL-7 and the vascular endothelial cell growth factor (VEGF). Those were significantly increased only when adaptive NK cells were co-cultured with HFF-TB40-BAC (Fig. 5a). In addition, adaptive NK cells cultured with target cells led to a significant increase of IL-5 secretion (mean: $19.89 \mathrm{pg} / \mathrm{ml}$, range: $3.11-46.73$ $\mathrm{pg} / \mathrm{ml}$ ). The interferon gamma-induced protein 10 (CXCL10) was only secreted at high levels (mean: $1930.16 \mathrm{pg} / \mathrm{ml}$ : range: $132.05-7380.23 \mathrm{pg} / \mathrm{ml}$ ), when adaptive NK cells were co-cultured with HFF-TB40-BAC. Under this condition, T cells had migrated to the bottom wells. T cells cultured with HFF-TB40-BAC showed increased secretion of IFN-a, (mean: $13.88 \mathrm{pg} / \mathrm{ml}$; range: 2.84-287.99 pg/ml), IL-7 (mean: $8.26 \mathrm{pg} / \mathrm{ml}$; range: $1.79-14.09$ $\mathrm{pg} / \mathrm{ml}$ ) and VEGF (mean: $22.28 \mathrm{pg} / \mathrm{ml}$; range: $0.82-37.92 \mathrm{pg} / \mathrm{ml}$ ) too, but at lower levels than in the combination of adaptive NK cells and HFF-TB40-BAC. Interestingly, the secretion of inhibitory cytokines like IL-1 receptor antagonist, IL-10 and IL-12p40 (Supplementary Fig. 1) were increased in the setting of adaptive NK cells stimulated with HFF-TB40-BAC as well. Statistical significance of the different cyto-/chemokines and VEGF is shown in Fig. 5b. Significant $p$ values are highlighted in gray.

\section{DISCUSSION}

Here we explored the immune responses of adaptive NK cells triggered by HCMV reactivation.

The current view is that HCMV infection is controlled by HCMVCTLs [20], but recently adaptive NK cells responsive to HCMV were described [21-23]. Redondo-Pachón et al. and Barron et al., strongly suggest an involvement of adaptive NK cells in the control of HCMV in kidney transplantation and in HSCT, respectively [24, 25]. Our data strongly support the observations of Barron et al. in patients early after HSCT and add to the current literature by including comparisons of immune reconstitution of HCMV-CTL and adaptive NK cells in the immunocompromised host.

The current opinion is that the NK cell compartment consists mainly of immature cells up to 6 months after HSCT [26]. We could show that mature, CD57-positive adaptive NK cells reconstitute early after HSCT in patients with HCMV-R (Fig. 1b). Adaptive NK cell numbers are also increased in healthy HCMV-seropositive individuals compared to seronegative ones, but are stable without HCMV-R [27]. Interestingly, we have shown that adaptive NK cells persist and further expand after resolution of HCMV reactivation in contrast to the retracting HCMV-CTL compartment (Figs. 1a and 2, respectively). This expansion did not affect the absolute cell counts of the total NK cell pool (Fig. 1c), suggesting that antigen/ pathogen recognition induces the maturation of adaptive NK cells after HCMV-R (Fig. 1d). This supports Cichocki et al., who observed 

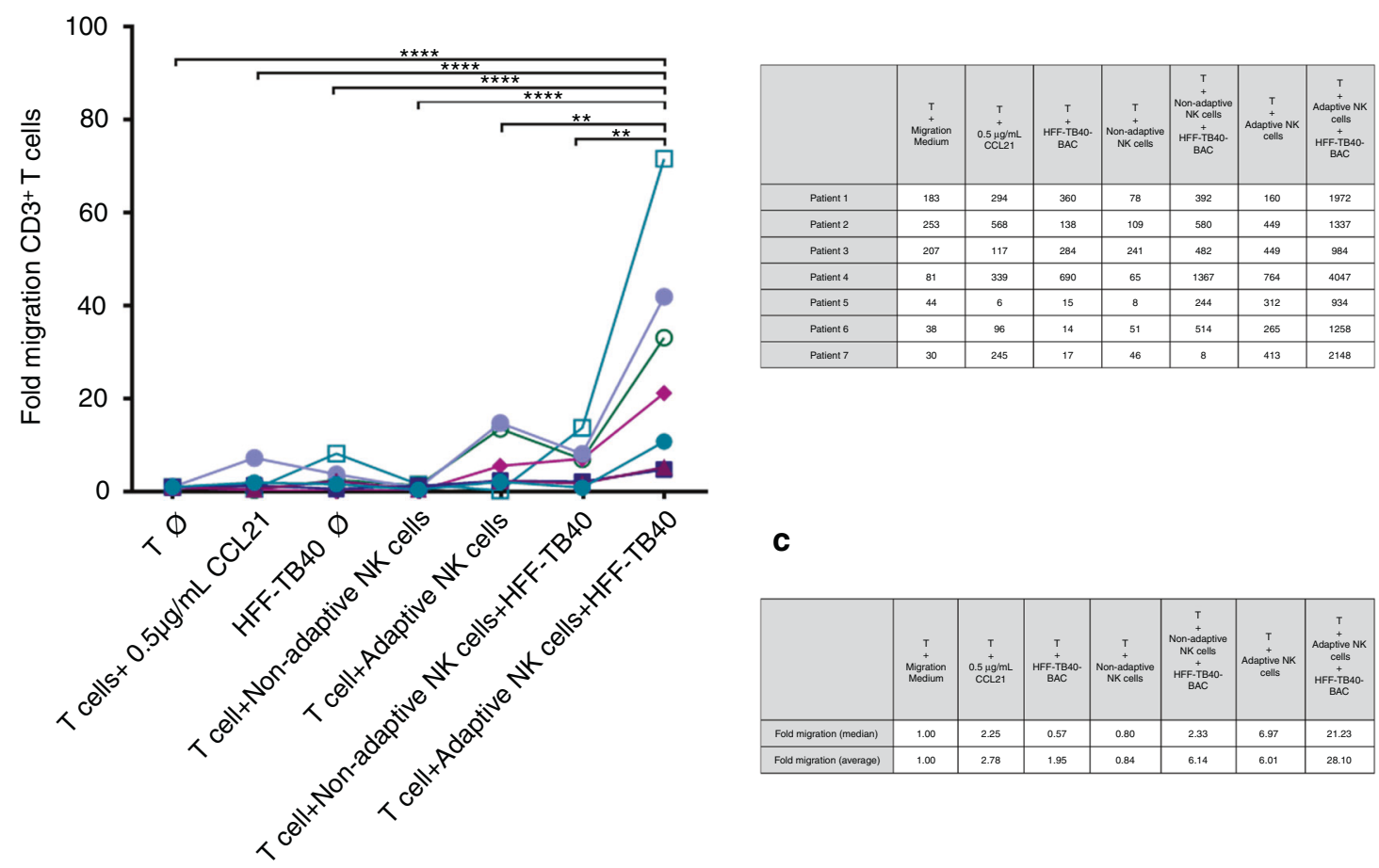

C

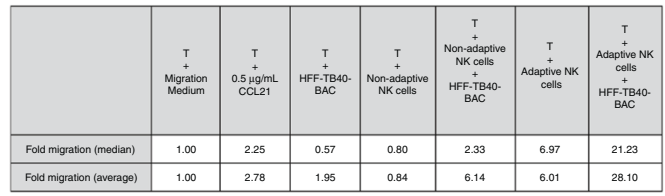

$\begin{array}{lll}\text { - Patient } 1 & \star \text { Patient } 2 & \text { - Patient } 3\end{array}$ - Patient 4

Fig. 4 Chemoattractant capacity of adaptive NK cells. a The fold migration of T cells in response to medium, the chemokine CCL21 (0.5 $\mu$ / $\mathrm{ml}$ ), HFF-TB40-BAC, non-adaptive NK cells, adaptive NK cells, non-adaptive NK cells and adaptive NK cells co-cultured with HFF-TB40-BAC is shown. b Summarized cell counts of migrated T cells. c Summarized fold migration (median and average) of $T$ cells. Statistical analysis represents two-way ANOVA with Tukey's multiple comparison. $n=7 ;{ }^{* *} p<0.01 ;{ }^{* * * *} p<0.0001$.

the expansion of adaptive NK cells after umbilical cord blood transplantation following reduced intensity conditioning [28, 29]. Patients who reactivated HCMV were divided into two groups regarding their early reconstitution of adaptive NK cells. Prior to clinical diagnose of HCMV-R, patients with absolute cell counts of adaptive NK cells equal or above $(\geq)$ the average were considered "Responders" and patients below this threshold $(<)$ were considered "Non-Responders". Only $25 \%$ of Responders had multiple HCMV-R, while $52 \%$ of Non-Responders developed several HCMV-R. In addition, the duration of HCMV-R in Responders was, on average, $\sim 10$ days shorter when compared to Non-Responders (duration of HCMV-R; Non-Responders: 30,075 days (range 6-125); Responders: 20,4 days (range 7-42)). Taken together, these results suggest that the expansion/ maturation of adaptive NK cells early on may influence HCMVimmunity, possibly controlling recurrent HCMV-R. HCMV can modulate the host immune response by downregulation of $\mathrm{MHC}$ class I molecules (MHC-I) circumventing $\mathrm{CD}^{+} \mathrm{T}$ cell recognition [30-32]. This leads in turn to a reduction of NK-inhibitory signals and to NK cell activation. Thus, adaptive NK cells may compensate for the lack of a T cell response due to down regulated MHC-I. On the other hand, HCMV can promote the surface expression of HLA$E$ thus engaging the NKG2 inhibitory receptor and HCMV expresses a viral homolog that binds to inhibitory receptors with higher affinity than MHC class I molecules [33-36], which could in turn down regulate NK cell responses. The expansion of adaptive NK cells in vivo after HCMV-R argues against this effect in the immunocompromised host (Fig. 1).

In addition, we could show that in vitro expanded, patientderived adaptive NK cells had an increased cytotoxicity toward
HFF-TB40-BAC leading to an increased LDH release (Fig. 3c). This demonstrates the capacity of expanded patient-derived adaptive NK cells to circumvent HCMV evasion mechanisms in vitro. In addition, NK cells are able to recruit other immune cells into the tumor/inflammation microenvironment [37]. To investigate the possible recruitment of $\mathrm{T}$ cells by adaptive NK cells in HCMVinfection setting, we performed transwell assays (Fig. 4). We could show that in vitro expanded adaptive NK cells co-cultured with HFF-TB40-BAC were able to promote $T$ cell recruitment to a significantly higher degree when compared to non-adaptive NK cells co-cultured with HFF-TB40-BAC, or adaptive NK cells cocultured with uninfected HFF. We assume that the cross-talk between the adaptive NK and autologous T cells may have complementary roles in the surveillance of HCMV. Our experiments required target cells for functional assays, which could not be retrieved from the patients. Fibroblast express the MHC class I chain-related protein A and B (MICA/B) on their cell surface, which activate NK cells through binding to NKG2D. We observed an increased expression of NKG2D in the NK subpopulation (data not shown), but we could also show that only the co-cultivation of the expanded adaptive NK cells with HFF-TB40-BAC led to significant increases in cytotoxicity or degranulation, when compared to adaptive NK cells co-cultured with uninfected HFF. One could argue that the virus used to infect HFF may not be comparable to HCMV strains found outside of laboratories. The TB40-BAC-BAC $\mathrm{KL7}^{-}$ SE-EGFP virus used here contains HCMV genes responsible for the inhibition of both T and NK cell thus is as close to HCMV as currently possible. Therefore, we postulate that the experimental set up had no major influence on the results presented here. It is also noteworthy that the assays can only be performed in vitro 
a

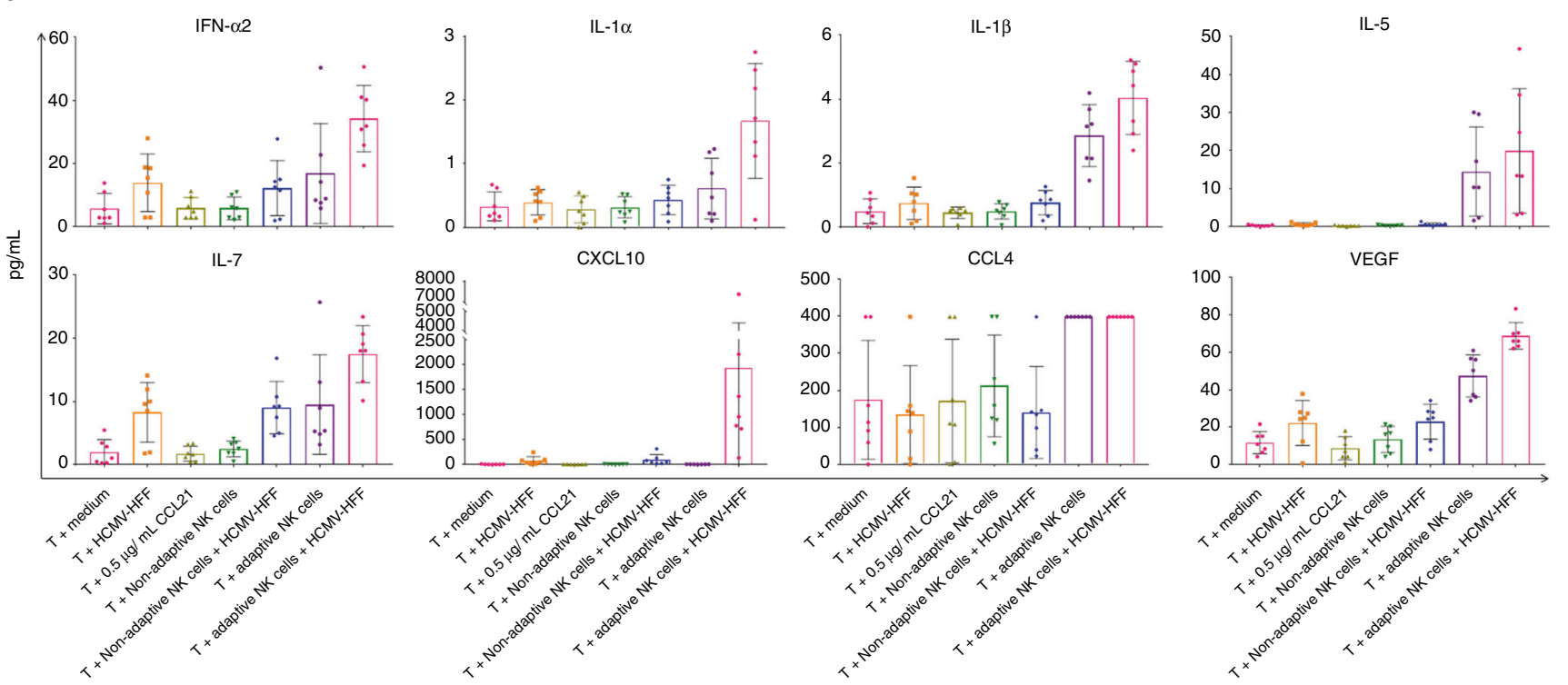

b

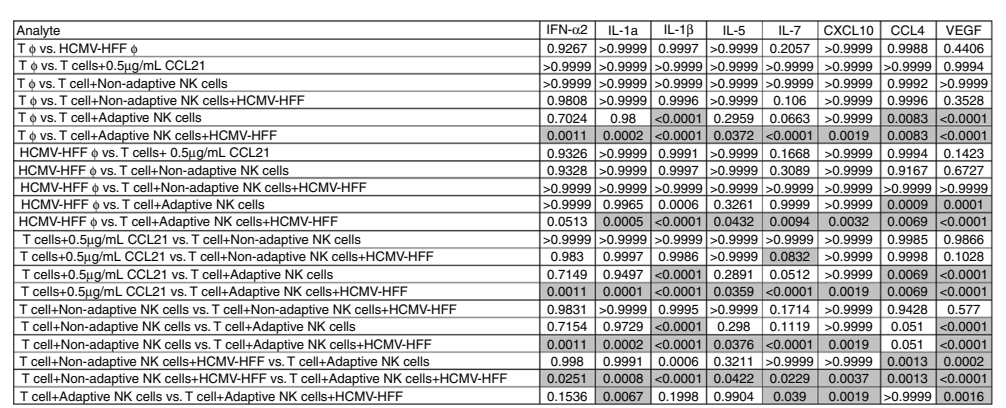

Fig. 5 Quantification of cytokines, chemokines and growth factors secreted in transwell assay in pg/ml. a Quantification of secreted cytokines and chemokines by T cells with medium; T cells with HFF-TB40-BAC; T cells with $0.5 \mu \mathrm{g} / \mathrm{ml} \mathrm{CCL21;} \mathrm{T} \mathrm{cells} \mathrm{with} \mathrm{non-adaptive} \mathrm{NK} \mathrm{cells;}$ T cells with both non-adaptive NK cells and HFF-TB40; T cells with adaptive NK cells and T cells with both adaptive NK cells and HFF-TB40. b Summarized $p$ values for each of the analytes. Statistical analysis represents one-way ANOVA with Tukey's multiple comparison. Mean and standard deviations are shown. Gray shaded cells represent statistically significant $p$ values; $n=7$. Below and above detectable levels were replaced by the lowest and highest standard reading, respectively.

and with expanded cells, due to the low frequency of these in immunosuppressed patients up to day +100 after HSCT. We could also show that activating cytokines and chemokines were excreted differentially in the transwell assays (Fig. 5), depending on the presence of adaptive NK cells stimulated with HFF-TB40BAC. IFN- $a$ as a signature anti-viral cytokine, was secreted by T cells stimulated with HFF-TB40-BAC, as expected [38], but at much lower levels than in the presence of adaptive NK cells. The chemokine CXCL10 directing $\mathrm{CXCR}^{+}$cells like macrophages, dendritic cells, NK and T cells to inflammation sites [39], was increased significantly only when adaptive NK cells co-cultured with HFF-TB40-BAC attracted T cells to the lower chambers of the transwell assay. CXCL10 is describe to induce apoptosis, to regulate cell growth and proliferation and to promote angiogenesis in infectious and inflammatory diseases [40]. The cytokine excretion pattern of the adaptive NK cells suggests an analogy to Th1 and Th2 cells. Th1 cells produce IFN- $\gamma$ and other proinflammatory cytokines also secreted by the adaptive NK cells when stimulated with HFF-TB40-BAC, while Th2 cells secrete regulatory cytokines like IL- 4 and IL-13 to down regulate immune responses. To overcome this regulatory effect Th1-cells excrete CXCL10 [40, 41]. Our data suggests that CXCL10 is produced by stimulated adaptive NK cells, thus possibly recruiting more of Th1 cells to the site of infection. The in vitro expanded adaptive
NK cells co-cultured with HFF-TB40-BAC and recruited T cells secreted also increased amounts of inhibitory cytokines like IL1RA, IL-10 and IL-12p40 (Supplementary Fig. 1). This may suggest a protection mechanism against over-activation and damage prevention by adaptive NK cells, while still maintaining an activating, pro-inflammatory and immune recruitment profile. These results suggest that in vitro expanded adaptive NK cells can recruit $T$ cells due to the increased secretion of several chemoand cytokines involved in immune modulation. Taken together our results of the in vivo monitoring of adaptive NK cells in patients with and without HCMV-R suggest a role of adaptive NK cells in the immune surveillance of HCMV in immune compromised hosts.

\section{PATIENTS AND METHODS}

\section{Patient cohort}

HCMV-seropositive patients $(\mathrm{R}+$ ) transplanted between 2016 and 2018 at the Hannover Medical School were eligible for inclusion in this observation study. The study was approved by the Institutional Review Board of the Hannover Medical School (protocol number: 2906). Patients and donors gave written informed consent in accordance with the declaration of Helsinki. Patients and donors were typed for 10 HLA alleles for exon $2+3$ for HLA-A, B, and C and for exon 2 for HLA-DRB1 and -DQB1 according to the current European Federation for Immunogenetics guidelines. Matched 
unrelated donors were identical for 10/10 HLA alleles with their recipients. All patients had a follow-up of at least 100 days after HSCT. Patient demographics and transplant procedures are summarized in Table 1. Clinical information on HCMV serostatus of donor (D) and recipient (R), number of patients with HCMV reactivation and day of HCMV reactivation is found on Table 2 .

\section{Monitoring of the NK cell immune reconstitution}

PB from HCMV-seropositive patients was collected and analyzed between day 0 and day +100 post-HSCT at intervals of 7-10 days or at the next visit at the out-patient clinic. Erythrocytes were lysed using VersaLyse solution (Beckman Coulter, Marseille, France) and resulting peripheral blood mononuclear cells (PBMCs) were stained for $30 \mathrm{~min}$ at room temperature (RT) with the antibodies summarized in Table 3.

Absolute numbers of adaptive NK cells per $\mu \mathrm{l}$ of whole blood were determined using fluorescent beads (FlowCount Fluorospheres ${ }^{\mathrm{T}} \mathrm{M}$, Beckman Coulter) and absolute cell number were calculated as previously described [42].

Nine samples (mean: 9; range: 4-12) per patient were analyzed. FACS analysis was performed using FlowJo Version 10 (Treestar, Ashland, USA).

\section{Monitoring of HCMV infection}

PB samples were collected and monitored for immune reconstitution of NK cells and HCMV-CTLs on days $25( \pm 10), 50( \pm 10)$ and $100( \pm 35)$ after transplantation, or weekly in case of HCMV reactivation. Erythrocytes were lysed and cells were fixed with VersaLyse (Beckman Coulter) with IOTest ${ }^{\circledR 3}$ fixative solution (Beckman Coulter). Samples were stained for $30 \mathrm{~min}$ at RT antibodies and/or tetramers are summarized in Table 4.

\section{Monitoring of HCMV reactivation}

PB samples of all patients were monitored weekly for pp65 antigen expression. HCMV reactivation was diagnosed when 5 or more pp65positive cells per $4 \times 10^{5}$ leukocytes were detected. Reactivation of HCMV was treated with ganciclovir as first line and foscarnet or others as second line therapy.

Table 3. Summarized flow cytometry panel used for the monitoring of adaptive NK cells.

\begin{tabular}{llll} 
Antibody & Conjugate & Clone & Company \\
\hline CD3 & PerCP & OKT3 & Biolegend \\
\hline CD14 & PerCP & HCD14 & Biolegend \\
\hline CD56 & APC-Cy7 & $5.1 H 11$ & Biolegend \\
\hline CD16 & PE-Cy7 & $3 G 8$ & Biolegend \\
\hline CD57 & FITC & HCD57 & Biolegend \\
\hline NKG2C & PE & 134591 & R\&D \\
\hline 7AAD & - & - & Biolegend \\
CD8 & AF700 & HIT8a & Biolegend \\
\hline NKG2D & BV421 & 1D11 & Biolegend \\
\hline NKp46 & BV605 & 9E2 & Biolegend
\end{tabular}

\section{Isolation of adaptive NK and T cells}

PBMCs from seven patients were isolated by density gradient centrifugation using standard Biocoll (Biochrom, Cambridge, United Kingdom) from PB samples of HCMV-seropositive patients, frozen and stored for analysis. For purification of NK and T cells, PBMCs were thawed and cultured overnight. Next, cells were stained for $10 \mathrm{~min}$ at $4{ }^{\circ} \mathrm{C}$ with recombinant human antibodies directed against: NKG2C (PE-Vio770 clone: REA205), CD57 (APC-Vio770 clone: REA769), CD3 (VioBlue clone: REA613) and CD56 (PE-Vio615 clone: REA196 all from Miltenyi, Bergisch Gladbach, Germany). Mature adaptive NK cells were sorted as $\mathrm{CD} 3^{-} \mathrm{CD}^{-} 6^{+} \mathrm{NKG} 2 \mathrm{C}^{+} \mathrm{CD} 57^{+}$; nonadaptive NK cells were sorted as NOT- $\mathrm{CD}^{-}{ }^{-} \mathrm{CD} 56^{+} \mathrm{NKG}_{2} \mathrm{C}^{+} \mathrm{CD} 57^{+}$and $T$ cells were sorted as $\mathrm{CD}^{+} \mathrm{CD}^{-} 6^{-}$. Adaptive NK cells were expanded in vitro as described below; non-adaptive NK and T cells cells were cryopreserved for later use.

\section{Ex vivo expansion of adaptive NK cells}

FACS-sorted $\mathrm{NKG}_{2} \mathrm{C}^{+} \mathrm{CD} 57^{+}$NK cells were co-cultured with irradiated $(25$ Gy) allogeneic PBMCs as feeder cells in Miltenyi NK-MACS medium. NKMACS was supplemented with $1 \%$ NK-MACS supplement, $1 \%$ Penicillin/ Streptomycin (Pen/strep), 5\% AB-serum, $500 \mathrm{IU} / \mathrm{ml}$ IL-2 (Miltenyi, Bergisch Gladbach, Germany), $10 \mathrm{ng} / \mathrm{ml}$ IL-15 (ImmunoTools, Friesoythe, Germany) and $1 \mu \mathrm{g} / \mathrm{ml}$ PHA (Invivogen, Toulouse, France) at $37^{\circ} \mathrm{C}$ and $5 \% \mathrm{CO}_{2}$ in a humidified atmosphere. On day 4 , half of the medium was removed and replaced with fresh medium supplemented with $1000 \mathrm{IU} / \mathrm{ml} \mathrm{IL-2}$ and $20 \mathrm{ng} /$ $\mathrm{ml} \mathrm{IL-15}$ and cells were continuously fed every 3 to 4 days. On day 14 of expansion, the phenotype of the cells was assessed by flow cytometry as described above.

\section{Virus propagation and titration}

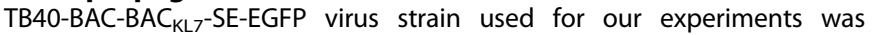
derived from human cytomegalovirus TB40/E was used to establish target cells for functional assays. TB40-BAC-BAC $\mathrm{KL}_{7}-\mathrm{SE}-\mathrm{EGFP}$ virus stocks were produced by propagation on human foreskin fibroblast (HFF) as described [43]. HFF were infected at $90-100 \%$ confluency with TB40-BAC-BAC $\mathrm{KL}_{\mathrm{KL}}-\mathrm{SE}-$ EGFP at an MOI of 0.1 and harvested at $100 \%$ cytopathic effect. Cellular debris was removed by centrifugation at $2800 \times g$ for $10 \mathrm{~min}$. Viral titers were determined by flow cytometric detection of EGFP expressing cells as described [44]. Briefly, $1 \times 10^{5} \mathrm{HFF}$ per well were seeded in triplicates into 48-well plates. DMEM supplemented with $10 \%$ fetal bovine serum and $1 \%$ Pen/Strep (DMEM 10\%) was added to the seeded cells together with virusdilutions ranging from $1 \times 10^{0}$ to $1 \times 10^{-2}$ and cells were incubated overnight. Virus inoculum was removed on the next day and cells were cultured for another $24 \mathrm{~h}$ in fresh DMEM 10\%. Cells were detached with a $0.05 \%$ trypsin, $0.02 \%$ EDTA solution (Sigma-Aldrich, Taufkirchen, Germany) and EGFP-positive cells were detected and counted by flow cytometry. The concentration of the virus stock was determined as infectious units per milliliter (IU/ml).

\section{Target cell preparation and NK cell degranulation assay}

Confluent HFF were infected at an MOI of 1 for $24 \mathrm{~h}$ and the infection rate was assessed by flow cytometry. Cells were thawed and cultured overnight prior to the degranulation assays. Expanded adaptive NK cells were cocultured with uninfected HFF, HCMV-infected HFF (HFF-TB40-BAC), K562 cells or without target cells as a control at effector: target ratios of 1:1; 2:1 and 5:1. CD107a (PE-Vio770, clone: REA792) was added to the cultures and

Table 4. Summarized flow cytometry panel used for the monitoring of HCMV-CTL.

\begin{tabular}{llll} 
Antibody/HLA-peptide (peptide sequence) & Conjugate & Clone & Company \\
\hline CD3 & PE-Cy7 & UCHT1 & Beckman Coulter, (Marseille, France) \\
\hline CD4 & PE & $13 B 8.2$ & Beckman Coulter, (Marseille, France) \\
CD8 & FITC & SFCl21Thy2D3 & Beckman Coulter, (Marseille, France) \\
\hline HLA-A*01:01 pp50 (VTEHDTLLY) & PE & $3 G 8$ & MBL International, (Woburn MA, USA) \\
\hline HLA-A*02:01 pp65 (NLVPMVATV) & FITC & HCD57 & MBL International, (Woburn MA, USA) \\
\hline HLA-A*02:01 IE1 (VLEETSVML) & PE & 134591 & MBL International, (Woburn MA, USA) \\
\hline HLA-A*24:02 pp65 (QYDPVAALF) & PE & - & MBL International, (Woburn MA, USA) \\
\hline HLA-B*07:02 pp65 (TPRVTGGGAM) & PE & HIT8a & MBL International, (Woburn MA, USA) \\
\hline HLA-B*08:01 IE1(ELRRKMMYM) & PE & $1 D 11$ & MBL International, (Woburn MA, USA) \\
\hline HLA-B*35:01 pp65 (IPSINVHHY) & PE & $9 E 2$ & MBL International, (Woburn MA, USA)
\end{tabular}


cells were incubated for $5 \mathrm{~h}$ at $37^{\circ} \mathrm{C}, 5 \% \mathrm{CO}_{2}$. Cells were washed and stained with antibodies directed against CD3 (Vio-Green, clone: REA613), NKG2C (APC, clone: REA205), CD57 (APC-Vio770, clone: REA769), CD56 (PerCP-Vio700, clone: REA196) and CD8 (VioBlue, clone: REA734) (Miltenyi, Bergisch Gladbach, Germany) for $10 \mathrm{~min}$ at $4{ }^{\circ} \mathrm{C}$.

\section{Lactate dehydrogenase (LDH) assay}

In vitro expanded adaptive NK cells were co-cultured with uninfected HFF, HFF-TB40-BAC or K562 cells (positive control) for $5 \mathrm{~h}$, cell-free supernatant was collected and analyzed using the Cytotoxic Detection Kit ${ }^{\text {Plus }}$ (LDH) (Roche, Basel, Switzerland) according to the manufacturer's recommendations. Briefly, $50 \mu \mathrm{l}$ of reaction mixture was added to $50 \mu \mathrm{l}$ of cell-free culture supernatant and incubated for $10 \mathrm{~min}$ at RT. Stop solution $(25 \mu \mathrm{l})$ was added and the absorbance was immediately measured at $490 \mathrm{~nm}$ in a TECAN Infinite M200 microplate reader (TECAN, Männedorf, Switzerland).

\section{Transwell assay for in vitro migration of $\mathbf{T}$ cells}

A transwell assay was used to assess the capacity of in vitro expanded adaptive NK cells to recruit autologous $\mathrm{CD}^{+} \mathrm{T}$ cells. Transwell permeable supports $6.5 \mathrm{~mm}$ insert, $5.0 \mu \mathrm{m}$ pore size polycarbonate membranes (Corning, Kaiserslautern, Germany) were used. $\mathrm{CD}^{+} \mathrm{T}$ cells were seeded at $5 \times 10^{5}$ cells $/ \mathrm{ml}$ and added to the top chamber of the transwell. The lower chambers were filled with $500 \mu \mathrm{l}$ of [1] migration medium, [2] CCL21 $(0.5 \mu \mathrm{g} / \mathrm{ml})$, [3] HFF-TB40-BAC, [4] non-adaptive NK cells, [5] non-adaptive NK cells co-cultured with HFF-TB40-BAC, [6] adaptive NK cells or [7] adaptive NK cells co-cultured with HFF-TB40-BAC. Effector to target ratio for NK cells co-cultured with HFF-TB40-BAC was 2:1 $\left(2.5 \times 10^{5} \mathrm{NK}\right.$ cells and $1.25 \times 10^{5}$ target cells). Cells were incubated at $37^{\circ} \mathrm{C}, 5 \% \mathrm{CO}_{2}$ for $8 \mathrm{~h}$ as described above. The supernatant was collected after $8 \mathrm{~h}$, centrifuged and frozen at $-80^{\circ} \mathrm{C}$ for further analysis. The bottom chamber was washed twice with PBS and cells were stained with anti-CD3 (PerCP clone: OKT3, BioLegend) and anti-CD8a (Alexa Flour 700 clone: HIT8a, BioLegend). Prior to acquisition, fluorescent counting beads (FlowCount Fluorospheres ${ }^{\mathrm{TM}}$, Beckman Coulter) were added to each sample.

\section{Quantification of cytokines and chemokines}

Cyto-/chemokine concentrations in cell-free supernatants were quantified by a multiplex protein array. Milliplex ${ }^{\circledR}$ Map Human Cytokine/Chemokine Magnetic bead panel (Merck Millipore, Darmstadt, Germany) was used according to the manufacturer's recommendations. Standards were reconstituted in deionized water, diluted from $10.000 \mathrm{pg} / \mathrm{ml}$ to $3.2 \mathrm{pg} / \mathrm{ml}$ and assay buffer alone was used as background control. Cell-free supernatant $(25 \mu \mathrm{l})$ was added to U-bottom 96-well plates and mixed with $25 \mu \mathrm{l}$ of assay buffer. Premixed beads $(25 \mu \mathrm{l})$ were added to each well and incubated shaking overnight at $4{ }^{\circ} \mathrm{C}$. Plates were washed twice with $200 \mu \mathrm{l}$ wash buffer. PE-labeled detection antibodies were added to each well and incubated for $1 \mathrm{~h}$ at RT. Beads were washed twice and suspended in $150 \mu \mathrm{l}$ sheath fluid and measured in the Luminex 200 Bioplex reader (Bio Rad, Hercules, CA, United States). Standard curves were calculated according to the five parameter logistic plot.

\section{Statistical analysis}

Statistical analysis was performed using Prism Version 7 (GraphPad Software Inc., California, USA). Tow-way ANOVA and Sidak's multiple comparison test, two-way ANOVA or one-way ANOVA with Tukey's multiple comparison test were used. The $p$ value $<0.05$ was considered statistically significant.

\section{REFERENCES}

1. Farhadfar N, Hogan WJ. Overview of the progress on haploidentical hematopoietic transplantation. World J Transplant. 2016;6:665.

2. Borchers S, Luther S, Lips U, Hahn N, Kontsendorn J, Stadler M, et al. Tetramer monitoring to assess risk factors for recurrent cytomegalovirus reactivation and reconstitution of antiviral immunity post allogeneic hematopoietic stem cell transplantation. Transpl Infect Dis. 2011;13:222-36.

3. Borchers S, Bremm M, Lehrnbecher T, Dammann E, Pabst B, Wölk B, et al. Sequential anti-cytomegalovirus response monitoring may allow prediction of cytomegalovirus reactivation after allogeneic stem cell transplantation. PLoS ONE. 2012;7:e50248.

4. Ogonek J, Varanasi PR, Luther S, Schweier P, Dammann E, Woelk B, et al. HLA matching influences the risk of recurrent reactivations and reconstitution of $C M V$ immunity after allogeneic stem cell transplantation. In 41st Annual Meeting of the European Society for Blood and Marrow Transplantation. 2015 March 22-25 2015:78.

5. Dolan A, Cunningham C, Hector RD, Hassan-Walker AF, Lee L, Addison C, et al. Genetic content of wild-type human cytomegalovirus. J Gen Virol. 2004;85:1301-12.

6. Boeckh M, Geballe AP. Cytomegalovirus: pathogen, paradigm, and puzzle. J Clin Invest. 2011;121:1673-80.

7. Picarda G, Benedict CA. Cytomegalovirus: shape-shifting the immune system. J Immunol. 2018;200:3881-9.

8. Ogonek J, Juric MK, Ghimire S, Varanasi PR, Holler E, Greinix H, et al. Immune reconstitution after allogeneic hematopoietic stem cell transplantation. Front Immunol. 2016;7:1-15.

9. Boeckh M, Nichols WG, Papanicolaou G, Rubin R, Wingard JR, Zaia J. Cytomegalovirus in hematopoietic stem cell transplant recipients: current status, known challenges, and future strategies. Biol Blood Marrow Transplant. 2003;9:543-58.

10. Hercend T, Takvorian T, Nowill A, Tantravahi R, Moningeon P, Anderson KC, et al. Characterization of natural killer cells with antileukemia activity following allogeneic bone marrow transplantation. Blood. 1986;67:722-8.

11. Zuo W, Zhao X. Natural killer cells play an important role in virus infection control: antiviral mechanism, subset expansion and clinical application. Clin Immunol. 2021;227:108727.

12. Drylewicz J, Schellens IMM, Gaiser R, Nanlohy NM, Quakkelaar ED, Otten $H$, et al. Rapid reconstitution of CD4 T cells and NK cells protects against CMV-reactivation after allogeneic stem cell transplantation. J Transl Med. 2016;14:1-9.

13. Gumá M, Angulo A, Vilches C, Gómez-Lozano N, Malats N, López-Botet M. Imprint of human cytomegalovirus infection on the NK cell receptor repertoire. Blood. 2004;104:3664-71.

14. Gumá M, Budt M, Sáez A, Brckalo T, Hengel H, Angulo A, et al. Expansion of CD94/ NKG2C + NK cells in response to human cytomegalovirus-infected fibroblasts. Blood. 2006;107:3624-31.

15. Hammer Q, Rückert T, Dunst J, Romagnani C. Adaptive natural killer cells integrate interleukin-18 during target-cell encounter. Front Immunol. 2018;8:1-9.

16. Foley B, Cooley S, Verneris MR, Pitt M, Curtsinger J, Luo X, et al. Cytomegalovirus reactivation after allogeneic transplantation promotes a lasting increase in educated NKG2C + natural killer cells with potent function. Blood. 2012;119:2665-74.

17. Sun JC, Beilke JN, Lanier LL. Adaptive immune features of natural killer cells. Nature. 2009;457:557-61.

18. Hammer $Q$, Rückert $T$, Borst EM, Dunst J, Haubner A, Durek $P$, et al. Peptidespecific recognition of human cytomegalovirus strains controls adaptive natural killer cells article. Nat Immunol. 2018;19:453-63.

19. Boeckh M. Current antiviral strategies for controlling cytomegalovirus in hematopoietic stem cell transplant recipients: prevention and therapy. Transpl Infect Dis. 1999;1:165-78.

20. Ljungman P, Hakki M, Boeckh M. Cytomegalovirus in hematopoietic stem cell transplant recipients. Hematol Oncol Clin North Am. 2011;25:151-69.

21. Björkström NK, Lindgren T, Stoltz M, Fauriat $C$, Braun $M$, Evander $M$, et al. Rapid expansion and long-term persistence of elevated NK cell numbers in humans infected with hantavirus. J Exp Med. 2011;208:13-21.

22. Béziat V, Dalgard $O$, Asselah T, Halfon P, Bedossa P, Boudifa A, et al. CMV drives clonal expansion of NKG2C + NK cells expressing self-specific KIRs in chronic hepatitis patients. Eur J Immunol. 2012;42:447-57.

23. Brunetta E, Fogli M, Varchetta S, Bozzo L, Hudspeth KL, Marcenaro E, et al. Chronic HIV-1 viremia reverses NKG2A/NKG2C ratio on natural killer cells in patients with human cytomegalovirus co-infection. AIDS. 2010;24:27-34.

24. Redondo-Pachón D, Crespo $M$, Yélamos J, Muntasell A, Pérez-Sáez MJ, PérezFernández $\mathrm{S}$, et al. Adaptive NKG2C + NK cell response and the risk of cytomegalovirus infection in kidney transplant recipients. J Immunol. 2017;198:94-101.

25. Barron MA, Gao D, Springer KL, Patterson JA, Brunvand MW, McSweeney PA, et al. Relationship of reconstituted adaptive and innate cytomegalovirus (CMV)-specific immune responses with CMV viremia in hematopoietic stem cell transplant recipients. Clin Infect Dis. 2009;49:1777-83.

26. $\mathrm{Yu}$ J, Freud AG, Caligiuri MA. Location and cellular stages of natural killer cell development. Trends Immunol. 2013;34:573-82.

27. López-Botet $M$, Muntasell $A$, Martínez-Rodríguez JE, López-Montañés $M$, CostaGarcía M, Pupuleku A. Development of the adaptive NK cell response to human cytomegalovirus in the context of aging. Mechanisms Ageing Dev. 2016;158:23-6.

28. Cichocki F, Taras E, Chiuppesi F, Wagner JE, Blazar BR, Brunstein C, et al. Adaptive NK cell reconstitution is associated with better clinical outcomes. JCI Insight. 2019;4:e125553.

29. Cichocki F, Cooley S, Davis Z, DeFor TE, Schlums H, Zhang B, et al. CD56 dim $\mathrm{CD} 57+\mathrm{NKG} 2 \mathrm{C}+\mathrm{NK}$ cell expansion is associated with reduced leukemia relapse after reduced intensity HCT. Leukemia. 2016;30:456-63. 
30. Yu D, Silva MC, Shenk T. Functional map of human cytomegalovirus AD169 defined by global mutational analysis. Proc Natl Acad Sci USA. 2003;100:12396-401.

31. Barnes PD, Grundy JE. Down-regulation of the class I HLA heterodimer and $\beta 2-$ microglobulin on the surface of cells infected with cytomegalovirus. J Gen Virol. 1992;73:2395-403.

32. Wiertz EJHJ, Jones TR, Sun L, Bogyo M, Geuze HJ, Ploegh HL. The human cytomegalovirus US11 gene product dislocates MHC class I heavy chains from the endoplasmic reticulum to the cytosol. Cell. 1996;84:769-79.

33. Braud VM, Allan DSJ, O'Callaghan CA, Soderstrom K, D'Andrea A, Ogg GS, et al. HLA-E binds to natural killer cell receptors CD94/NKG2A, B and C. Nature. 1998;391:795-9.

34. Lee $N$, Llano $M$, Carretero $M$, Ishitani $A$, Navarro F, López-Botet $M$, et al. HLA-E is a major ligand for the natural killer inhibitory receptor CD94/NKG2A. Proc Natl Acad Sci USA. 1998;95:5199-204.

35. Beck S, Barrell BG. Human cytomegalovirus encodes a glycoprotein homologous to MHC class-I antigens. Nature. 1988:331:269-72.

36. Chapman TL, Heikema AP, Bjorkman PJ. The inhibitory receptor LIR-1 uses a common binding interaction to recognize class I MHC molecules and the viral homolog UL18. Immunity. 1999;11:603-13.

37. Böttcher JP, Bonavita E, Chakravarty P, Blees H, Cabeza-Cabrerizo M, Sammicheli $S$, et al. NK Cells stimulate recruitment of $\mathrm{CDC1}$ into the tumor microenvironment promoting cancer immune control. Cell. 2018;72:1022-37.e14.

38. Lun MT, Lorino G, Gaeta A, Dessy P, Cipriani P, Filadoro $P$, et al. In vitro interferon production: induction in human leukocytes after exposure to cytomegalovirus antigens. Microbiologica. 1986;9:399-404.

39. Romagnani S. The Th1/Th2 paradigm. Immunol Today. 1997;18:263-6.

40. Liu M, Guo S, Hibbert JM, Jain V, Singh N, Wilson NO, et al. CXCL10/IP-10 in infectious diseases pathogenesis and potential therapeutic implications. Cytokine Growth Factor Rev. 2011;22:121-30.

41. Berger A. Th1 and Th2 responses: what are they? BMJ. 2000;321:424.

42. Neudoerfl C, Mueller BJ, Blume C, Daemen K, Stevanovic-Meyer M, Keil J, et al. The peripheral NK cell repertoire after kidney transplantation is modulated by different immunosuppressive drugs. Front Immunol. 2013;4:1-14.

43. Wu Z, Sinzger C, Reichel JJ, Just M, Mertens T. Natural killer cells can inhibit the transmission of human cytomegalovirus in cell culture by using mechanisms from innate and adaptive immune responses. J Virol. 2015;89:2906-17.

44. Basílio-Queirós D, Venturini L, Sampaio KL, Sinzger C, Weissinger EM. Fast and efficient titration of human cytomegalovirus stocks with a self-excisable bacterial artificial chromosomes cassette by flow cytometry. Hum Gene Ther Methods. 2019;30:122-6

\section{ACKNOWLEDGEMENTS}

We thank Prof. Dr. Christian Sinzger for providing TB40-BAC $\mathrm{KL}_{7}-\mathrm{SE}-\mathrm{EGFP}$ virus and Jana Keil and Kerstin Beushausen for excellent technical assistance. We would also like to acknowledge the assistance of the Cell Sorting Core Facility of the Hannover Medical School, in part supported by Braukmann-Wittenberg-Herz-Stiftung and Deutsche Forschungsgemeinschaft.

\section{AUTHOR CONTRIBUTIONS}

DBQ planned the study, performed and oversaw the experiments, wrote the paper and discussed data; LV performed experiments and contributed to the paper, SLW provided excellent technical assistance. ED provided excellent documentation and generated the data base for all patients and follow up; AG and MS provided patients, discussed results and contributed to the writing; CSF performed experiments and discussed the data, EMW contributed to experiments, patients and the writing of the paper.

\section{FUNDING}

Open Access funding enabled and organized by Projekt DEAL.

\section{COMPETING INTERESTS}

The authors declare no competing interests.

\section{ADDITIONAL INFORMATION}

Supplementary information The online version contains supplementary material available at https://doi.org/10.1038/s41409-022-01603-y.

Correspondence and requests for materials should be addressed to Débora BasílioQueirós.

Reprints and permission information is available at http://www.nature.com/ reprints

Publisher's note Springer Nature remains neutral with regard to jurisdictional claims in published maps and institutional affiliations.

(i) Open AccessThis article is licensed under a Creative Commons Attribution 4.0 International License, which permits use, sharing, adaptation, distribution and reproduction in any medium or format, as long as you give appropriate credit to the original author(s) and the source, provide a link to the Creative Commons licence, and indicate if changes were made. The images or other third party material in this article are included in the article's Creative Commons licence, unless indicated otherwise in a credit line to the material. If material is not included in the article's Creative Commons licence and your intended use is not permitted by statutory regulation or exceeds the permitted use, you will need to obtain permission directly from the copyright holder. To view a copy of this licence, visit http://creativecommons. org/licenses/by/4.0/.

(c) The Author(s) 2022 